\title{
Far-zone Resonant Energy Transfer in X-Ray Photoemission as a Structure Determination Tool
}

Denis Céolin, ${ }^{, \dagger \dagger}$ Jean-Pascal Rueff ${ }^{\dagger}$ Andrey Zimin,,$+{ }^{\dagger}$ Paul Morin, ${ }^{\dagger}$ Victor

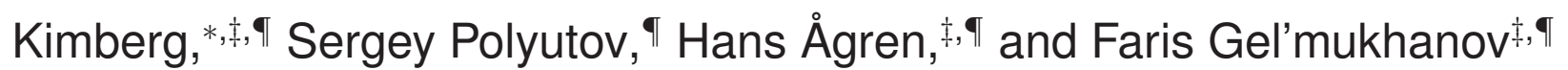

†Synchrotron SOLEIL, l'Orme des Merisiers, Saint-Aubin, BP 48, 91192 Gif-sur-Yvette Cedex, France

$\ddagger$ Theoretical Chemistry and Biology, School of Biotechnology, Royal Institute of Technology, S-106 91 Stockholm, Sweden

【Institute of Nanotechnology, Spectroscopy and Quantum Chemistry, Siberian Federal University 660041 Krasnoyarsk, Russia

E-mail: denis.ceolin@synchrotron-soleil.fr; kimberg@kth.se 


\begin{abstract}
Near-zone Förster resonant energy transfer is the main effect responsible for excitation energy flow in the optical region and is frequently used to obtain structural information. In the hard X-ray region the Förster law is inadequate because the wavelength is generally shorter than the distance between donors and acceptors and, hence, far-zone resonant energy transfer (FZRET) becomes dominant. We demonstrate the characteristics of X-ray FZRET and its fundamental differences with the ordinary near-zone resonant energy transfer process in the optical region by by recording and analyzing two qualitatively different systems - high-density $\mathrm{CuO}$ polycrystalline powder and $\mathrm{SF}_{6}$ diluted gas. We suggest a method to estimate geometrical structure using X-ray FZRET employing as a ruler the distance dependent shift of the acceptor core ionization potential induced by the Coulomb field of the core-ionized donor.
\end{abstract}

\title{
Graphical TOC Entry
}

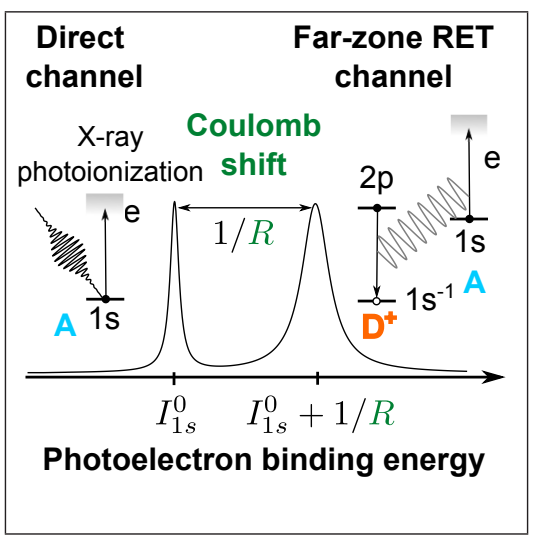


Resonant energy transfer (RET) occurs when an electronically excited donor transfers energy to an acceptor. The RET process constitutes the main energy exchange mechanism in systems ranging from color centers in crystalline materials to photosynthetic light-harvesting machineries and fluorescent proteins. ${ }^{1-3}$ In the optical region, RET is governed by the near-zone Förster mechanism $^{4,5}$ and provides an analytical tool, the "spectroscopic ruler", 2,6 to estimate the donoracceptor separation between coupled chromophores. However, as we show here for the first time, RET in the hard X-ray region is essentially a far-zone phenomenon where the finite propagation time of the photons is of crucial importance due to the small wavelength of the X-ray photons. The RET process is mediated by photon exchange between a donor $(D)$ and an acceptor $(A)$, where the finite propagation time of the photons results in a retardation effect described by the parameter $k R$, where $k$ is the wavenumber of the photon and $R$ is the donor-acceptor separation. The original theory of RET was developed by Avery, ${ }^{7}$ and by Gomberoff and Power ${ }^{8}$ based on general quantum electrodynamics (QED). In later works ${ }^{9,10}$ the RET rate was identified as $\gamma(R) \propto R^{-6}\left(1+\frac{1}{3}\left[(k R)^{2}+(k R)^{4}\right]\right)$, which behaves as $\gamma(R) \propto R^{-2}$ for large $R$ and is vastly different from the Förster rate ref-6 $^{2, R}(R) R^{-6}$. We note that QED and classical electrodynamics give the same $R$-dependence of the transfer rate. ${ }^{11}$ Contributions to the transfer rate arising from the near-, middle- and far-zone terms decay with $R$ as $1 / R^{6},(k R)^{2} / R^{6} \propto k^{2} R^{-4}$ and $(k R)^{4} / R^{6} \propto k^{4} R^{-2}$, respectively.

Retardation effects can be safely neglected in the optical region due to short propagation distances according to the well-known Förster near-zone RET rate $\gamma(R) \propto R^{-6}$. However, in the X-ray region the photon wavelength is below typical donor-acceptor separations; for instance $k R=8.6$ for the first coordination sphere in $\mathrm{CuO}$ at $\mathrm{Cu}-\mathrm{K} \alpha$ radiation. Contrary to the optical region, where the Förster RET mechanism is valid, the RET process in the X-ray region is essentially a far-zone RET (FZRET) process. We report here experimental observations of this process in the X-ray region, by studying high energy X-ray FZRET from a core-ionized donor atom to an acceptor atom. In order to use the FZRET process as an analytical tool to measure donor-acceptor separations we need an appropriate spectroscopic label of the acceptor atom. This label arises nat- 
urally in the two-step core ionization of the acceptor atom $A$ shown schematically on Fig. 1(a,b). The ionization of the core level of the donor atom $D$ by an incoming X-ray photon (Fig. 1(a)), leads to a sudden increase in the Coulomb field by the positively charged donor $1 s$ core-hole $D^{+}$. In the spirit of the ESCA model ${ }^{12}$ (ESCA - Electron Spectroscopy for Chemical Analysis) this added charge shifts the ionization potential $I_{1 s}^{0}$ of the $1 s$ level of the acceptor atom $I_{1 s}=I_{1 s}^{0}+\Delta$ by an amount $\Delta=q / R$. Within the realm of this model and with the donor atoms starting from the third row the FZRET process gives the possibility to record the $\mathrm{K}$ photoelectron lines of lighter atoms of the molecule and to estimate the donor-acceptor distances $R$ using simple relation between $R$ and the shift. We note that despite that the ESCA model neglects core hole relaxation induced screening of the charge distributions, it has been shown applicable to a wide variety of compounds, including $\mathrm{SF}_{6}$ and other fluorine compounds. ${ }^{12}$ Let us also note that there is no Coulomb shift $\Delta$ in multi-atom resonant photoemission (MARPE), ${ }^{13,14}$ where core-ionization of the donor is absent. The energy $\hbar \omega_{D}$ released in the course of the core-hole annihilation $2 p \rightarrow 1 s$ is resonantly transferred to the acceptor atom, ejecting its core-electron to the continuum (Fig. 1(b)). We measure the energy of the ejected electron $E_{\mathrm{el}}$ and compute its binding energy as $\mathrm{BE}=\hbar \omega_{D}-E_{\mathrm{el}}-\phi$, where $\phi$ is the work function of the material. The element-specific resonant value $I_{1 s}=\mathrm{BE}$ of the binding energy determines unambiguously the type of the acceptor and provides an estimate for the donor-acceptor separation from $\Delta=I_{1 s}-I_{1 s}^{0}=q / R$ as $R=q / \Delta$. Note that $I_{1 s}^{0}$ should be measured independently by direct ionization of the acceptor.

The FZRET signal is collected from all acceptor atoms accessible to the donor, as follows from the expression for the spectral density of the FZRET rate $\gamma(\mathrm{BE})$ :

$$
\gamma(\mathrm{BE})=\Gamma_{\mathrm{rad}} \sum_{R}\left(\frac{R_{F}}{R}\right)^{6} \frac{\Gamma\left(1+\frac{1}{3}\left[(k R)^{2}+(k R)^{4}\right]\right)}{\pi\left[\left(\mathrm{BE}-\left(I_{1 s}^{0}+\frac{1}{R}\right)\right)^{2}+\Gamma^{2}\right]} e^{-R / l},
$$

where the sum runs over all acceptor-donor pairs. Here $l$ is the attenuation length $(l \approx 27 \mu m$ for $\hbar \omega_{D}=8 \mathrm{keV}^{15,16}$ for $\mathrm{CuO}$ ), $\Gamma_{\text {rad }}$ is the radiative rate (typically about $1-10 \mathrm{fs}^{-1}$ ), $\Gamma$ is the total decay rate of the core ionized donor atom, and $R_{\mathrm{F}}$ is the Förster radius. Note that there is no 
interference between core-excited states localized on different donor atoms in contrast to resonant X-ray Raman scattering ${ }^{17,18}$ and MARPE. ${ }^{19}$ In the case of solid samples acceptors are taken from a surface layer with a thickness of about the electron inelastic mean free path $(\approx 100 \mathrm{~nm})$. In the optical region the main contribution to the RET rate $\gamma(\mathrm{BE})$ originates from the nearest coordination spheres because the near-zone Förster RET rate quickly drops down as $1 / R^{6}$. The picture changes drastically in the X-ray region where the far-zone term $k^{4} / R^{2}$ dominates (Fig.1(c)) and significantly enhances the signal from the outer coordination spheres, where the number of acceptors grows as an area of a sphere $\propto R^{2}$ and reduces the decay of the FZRET rate by factor $R^{-2} e^{-R / l}$. The weak decrease of the FZRET rate gives a unique opportunity to distinguish the dominant contributions through the shift of the acceptor ionization potentials. One can expect a large shift $\Delta \approx 1 / R_{0} \approx 10 \mathrm{eV}$ when the nearest neighbors within the first coordination sphere $R_{0} \approx 0.15 \mathrm{~nm}$ contribute the most, and a negligible shift when the outer coordination spheres $R \gg 5 \mathrm{~nm}$ dominate. To clarify this question we experimentally study two qualitatively different systems: polycrystalline $\mathrm{CuO}$ powder and diluted $\mathrm{SF}_{6}$ gas at low pressure $\left(10^{-3} \mathrm{mbar}\right)$.

Experiments were conducted at the GALAXIES high brightness hard X-ray beamline (photon energy in the range 2.4-12 keV) of the SOLEIL synchrotron (France) coupled with newly operational HAXPES endstation with high transmission and resolution. ${ }^{20,21}$ Our first studied system is a polycrystalline powder of $\mathrm{CuO}$ semiconductor, where the FZRET process occurs between a $\mathrm{Cu}$ donor and an $\mathrm{O}$ acceptor. The sample was prepared as a pellet of high purity powder. Measurements were done at $1^{\circ}$ incidence angle compared with the photon propagation axis. The photon polarization axis is collinear to the spectrometer lens axis and at $89^{\circ}$ of the $\mathrm{CuO}$ sample surface. This sample is characterized by a high density of oxygen acceptors available for a given copper donor. We do not expect to observe a spectral shift (i.e. $\Delta=0$ ) because the dominant contribution is coming from the outer coordination spheres with large $\mathrm{R}$. The second system is the $\mathrm{SF}_{6}$ gas, where resonant energy transfer occurs between a $\mathrm{S}$ donor and a $\mathrm{F}$ acceptor. The photons go through a gas cell, in which the pressure was kept at about $10^{-3}$ mbar, corresponding to a concentration of molecules of about $n=2.4 \times 10^{13} \mathrm{~cm}^{-3}$ at $\mathrm{T}=300 \mathrm{~K}$. This corresponds to a pressure in 

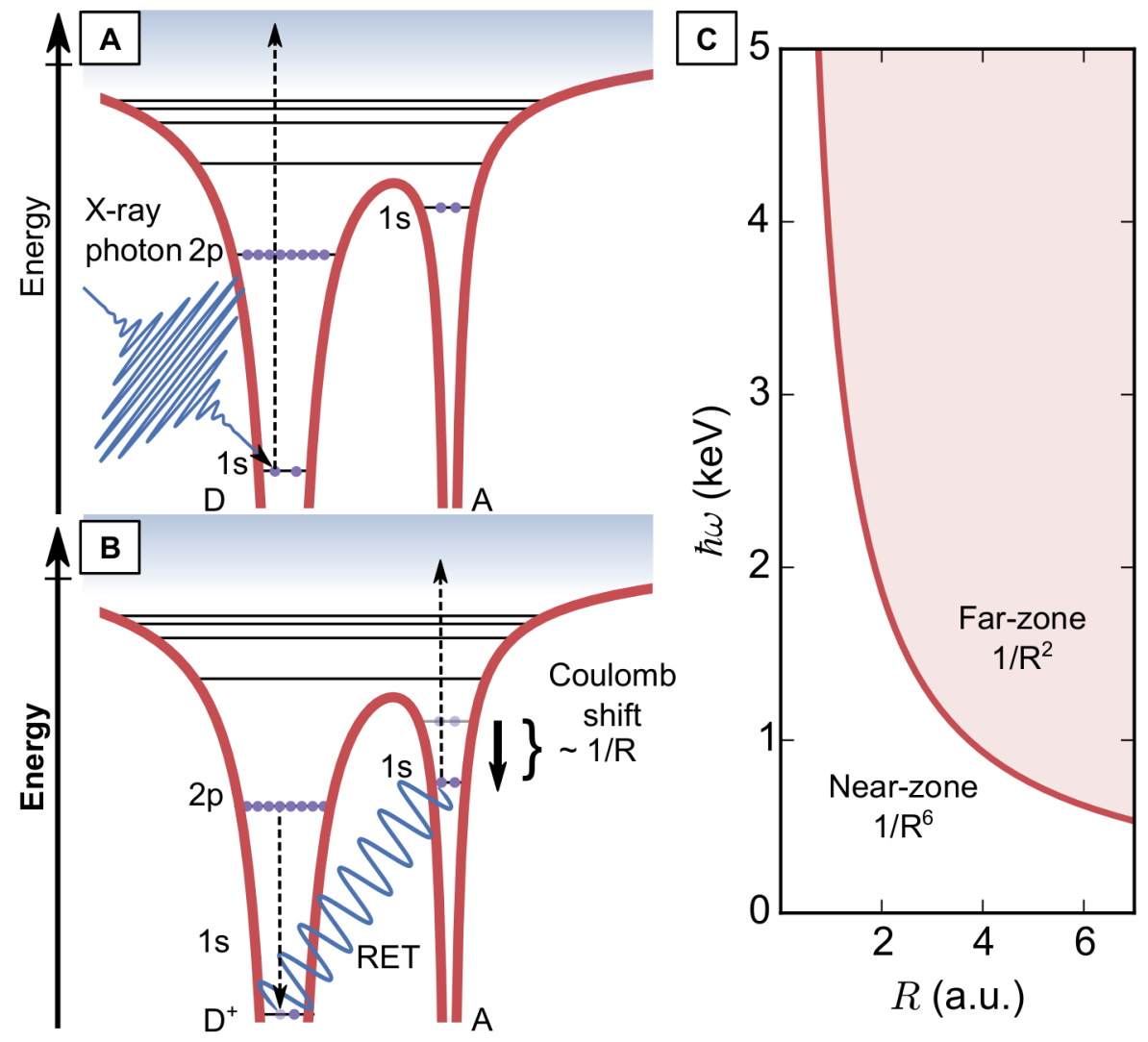

Figure 1: Scheme of resonant energy transfer following core ionization of the donor. (A) Core ionization of the donor atom (D) by X-ray photon forms a positively charged core hole. The donor core hole induces a Coulomb shift $\propto 1 / R$ of the acceptor ionization potential (B) and the energy released in the $2 p \rightarrow 1 s$ donor relaxation is resonantly transferred to the acceptor, ejecting its core electron to the continuum. (C) Near and far zone regions in $(\omega, R)$ plane. Red area above the $\hbar \omega=c / R$ line shows a region where the far-zone RET term $k^{4} / R^{2}$ dominates the transfer rate. The energy values presented on the panels $\mathrm{A}$ and $\mathrm{B}$ correspond to $\mathrm{SF}_{6}$. 
the SCIENTA spectrometer chamber of about $10^{-5}$ mbar. The geometry of the cell is a cylinder of $1 \mathrm{~cm} \times 1 \mathrm{~cm}$ (length $\mathrm{x}$ diameter) equipped with $1 \mathrm{~mm} \times 1 \mathrm{~cm}$ slits in the direction of the spectrometer lens axis to collect the photoelectrons. The mean distance between molecules in this cell, $R_{c}=(3 /(4 \pi n))^{1 / 3}=2.1 \times 10^{3} \AA$, is much larger than the bond length $(\sim 1 \AA)$. This is why the intramolecular RET rate exceeds at least two orders of magnitude the extramolecular RET rate (see the Supporting Information for explanations and computational details), resulting in a large Coulomb shift $\Delta \approx 11 \mathrm{eV}$.

We measured the FZRET signal for both systems, $\mathrm{CuO}$ and $\mathrm{SF}_{6}$, at two different excitation energies far above the core-ionization thresholds, and found profiles to be identical independently of the incoming photon energy in order to eliminate possible artifacts and post-collision interaction (PCI) effects. ${ }^{22}$ The position of the FZRET resonance in the binding energy scale is the key parameter responsible for the accuracy of our measurements. The energy of this peak is defined with the accuracy $\pm 1.2 \mathrm{eV}$ for $\mathrm{CuO}$ and $\pm 1.0 \mathrm{eV}$ for $\mathrm{SF}_{6}$. For $\mathrm{SF}_{6}$ the measurements were performed at 2700 and $2800 \mathrm{eV}$ while for $\mathrm{CuO}$ they were performed at 9100 and $9400 \mathrm{eV}$. For this latter system, we measured the $\mathrm{Cu} 1 \mathrm{~s}, \mathrm{Cu} 2 \mathrm{p}, \mathrm{O} 1$ s core lines, valence band positions and X-ray absorption spectrum at the $\mathrm{Cu}$ K-edge for calibration purpose. Based on this calibration, the $\mathrm{O}$ 1s ionization potential and average work function are found to be $I . P .(O 1 s)=529.4 \mathrm{eV}$ and $\phi=4.9 \mathrm{eV}$, respectively. The $\mathrm{Cu} \mathrm{K}$-edge position is measured at $8986 \mathrm{eV}$ and is shifted by $\approx 6 \mathrm{eV}$ relative to the pure crystalline $\mathrm{Cu}$ position at $8980.48 \mathrm{eV} .{ }^{23}$ The $\mathrm{K}_{\alpha 1}$ and $\mathrm{K}_{\alpha 2}$ lines are found at $8047.8 \mathrm{eV}$ and $8027.8 \mathrm{eV}$, respectively, in perfect agreement with the values $8047.82 \mathrm{eV}$ and $8027.84 \mathrm{eV}$ given in reference. ${ }^{24}$ For $\mathrm{SF}_{6}$, the energy transfer from $\mathrm{S}$ to $\mathrm{F}$ is obtained by exciting sulphur above its K-shell ionization threshold located at $2490.1 \mathrm{eV} .{ }^{25}$ The FZRET peak corresponds to the ionization of the F 1s orbital (I.P.=694.6eV ${ }^{26}$ ) by the unresolved S $K_{\alpha 1,2}$ doublet which has the energy of $2309.3 \mathrm{eV} .{ }^{27}$ To test the influence of pressure, we measured the spectra at two different pressures $\left(5 \times 10^{-6} \mathrm{mbar}, 1.5 \times 10^{-5} \mathrm{mbar}\right)$. The results show no difference within the error bar.

For the $\mathrm{CuO}$ system, we prove that we indeed observe RET from $\mathrm{Cu}$ to $\mathrm{O}$ on Fig. 2. The $\mathrm{O}$ $1 s$ lines occur only when the excitation energy exceeds the minimal energy $8986 \mathrm{eV}$ necessary to 
open the $\mathrm{Cu} \mathrm{K} \mathrm{K}_{\alpha}$ emission channel. These lines are located at $7513.5 \mathrm{eV}$ and $7493.5 \mathrm{eV}$, and copy the shape of the $\mathrm{Cu} \mathrm{K}_{\alpha 1,2}$ lines known to be at $8047.8 \mathrm{eV}$ and $8027.8 \mathrm{eV} .{ }^{28}$ Experimental FZRET profiles for $\mathrm{CuO}$ and $\mathrm{SF}_{6}$ are shown in Fig. 3(a) and Fig. 3(b), respectively. For the $\mathrm{CuO}$ system, the oxygen FZRET peak is not shifted relative to the direct photoionization peak. On the contrary, for $\mathrm{SF}_{6}$ there is a $10.9 \mathrm{eV}$ shift of the fluorine FZRET peak. To explain the absence of the shift in the $\mathrm{CuO}$ case, we computed the RET rate (see equation 1) from all acceptor-donor pairs inside a spherical volume defined by the cut-off radius $R_{c}$ around the acceptor O atom (Fig. 4(a)). When we take only four nearest neighbors into account within $R_{c}=0.21 \mathrm{~nm}$, we clearly see that the RET signal is shifted by $7.4 \mathrm{eV}$. If we include donors up to $R_{c}=0.50 \mathrm{~nm}$, a second peak shifted by $3.5 \mathrm{eV}$ emerges, as well as a transition region between the two peaks. By further expanding the cut-off radius up to $5.0 \mathrm{~nm}$, we see that the dominant contribution becomes almost unshifted. We get convergence at $R_{c}=50 \mathrm{~nm}$ where the RET signal is represented by a single unshifted peak. The absence of the shift is a direct evidence that the RET profile is formed by remote shells where $\Delta(R) \rightarrow 0$. It is interesting to compare our expectations with the Förster RET theory (equation 1 with $k R=0$ ). Förster RET theory contradicts experiment and predicts the shift of the signal (Fig. 4(b)) without any contributions from the acceptors located farther than $1 \mathrm{~nm}$ from the donor atom due to the fast $R^{-6}$ decay.

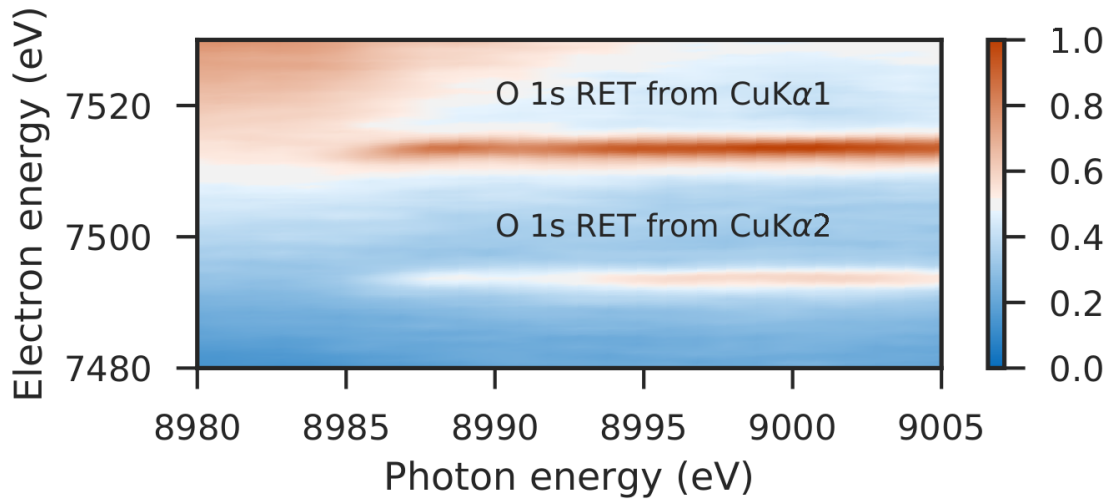

Figure 2: Experimental evidence for RET in X-ray photoemission spectrum for solid CuO. 2D map shows that $\mathrm{O} 1 s$ peaks are formed only when photon energy exceeds $\mathrm{Cu}$ core-ionization threshold at $8986 \mathrm{eV}$. The excitation energy $\hbar \omega$ is scanned across the copper $1 s$ edge and we measure the kinetic energy of the $\mathrm{O} 1 s$ electrons emitted due to RET in the course of $\mathrm{Cu} K_{\alpha_{1,2}}$ decay. 


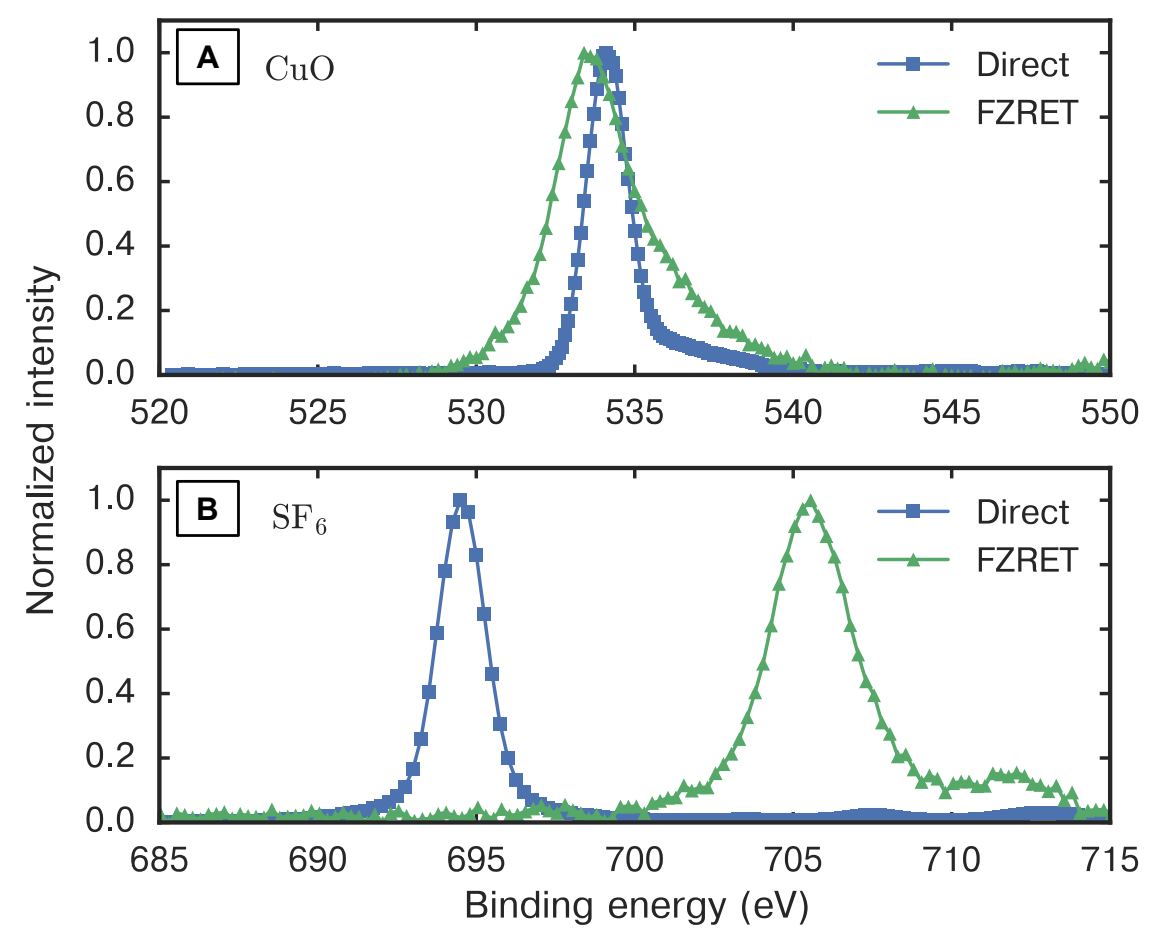

Figure 3: Normalized to maximum experimental FZRET profiles and direct photoelectron spectra of $\mathrm{O} 1 \mathrm{~s}$ in solid $\mathrm{CuO}(\mathrm{A})$ and $\mathrm{F} 1 \mathrm{~s}$ in the diluted $\mathrm{SF}_{6}$ gas (B). (A) The shift of the O1s line is absent (within the accuracy of the measurements $\pm 1.2 \mathrm{eV}$ ) in condensed $\mathrm{CuO}$, where the energy is transferred mainly to remote oxygen atoms (see Fig.4A). FZRET peak was measured after excitation of $\mathrm{Cu} 1 s$ by $9400 \mathrm{eV}$ photons. The asymmetry of FZRET profile for $\mathrm{CuO}$ is caused by the asymmetry of the $\mathrm{Cu} \mathrm{K}$ line explained earlier (see ref. ${ }^{24}$ and references therein). (B) The $10.9 \mathrm{eV}$ shift of the $F 1 s$ line is large because RET profile in diluted $\mathrm{SF}_{6}$ is formed mainly due to the intramolecular RET. FZRET peak was measured after excitation of $\mathrm{S} 1 s$ by $2700 \mathrm{eV}$ photons. One should notice that the intensity of the FZRET signal is much weaker than the intensity of direct ionization (see Figure 1 in Supporting Information).

In contrast to the $\mathrm{CuO}$ system, the $\mathrm{SF}_{6}$ diluted gas shows a substantially shifted signal in the experiment. The density of the acceptor atoms is high only in the first coordination sphere of the donor atom, so we observe a signal shifted by $10.9 \mathrm{eV}$ emerging from the intramolecular FZRET process and allowing us to estimate the interatomic separation in the $\mathrm{SF}_{6}$ molecule as $R_{\mathrm{SF}}=1.32 \AA$. This can be compared with the reference value $1.56 \AA{ }^{29}$ The main reasons for the deviation of $R$ is the rather large error in measurements of the shift $\Delta( \pm 1 \mathrm{eV}$ ) (see Supporting Information) and the neglect of screening relaxation in the simple ESCA model. The contribution of the remote acceptors (extramolecular) is negligible in this case due to geometrical 


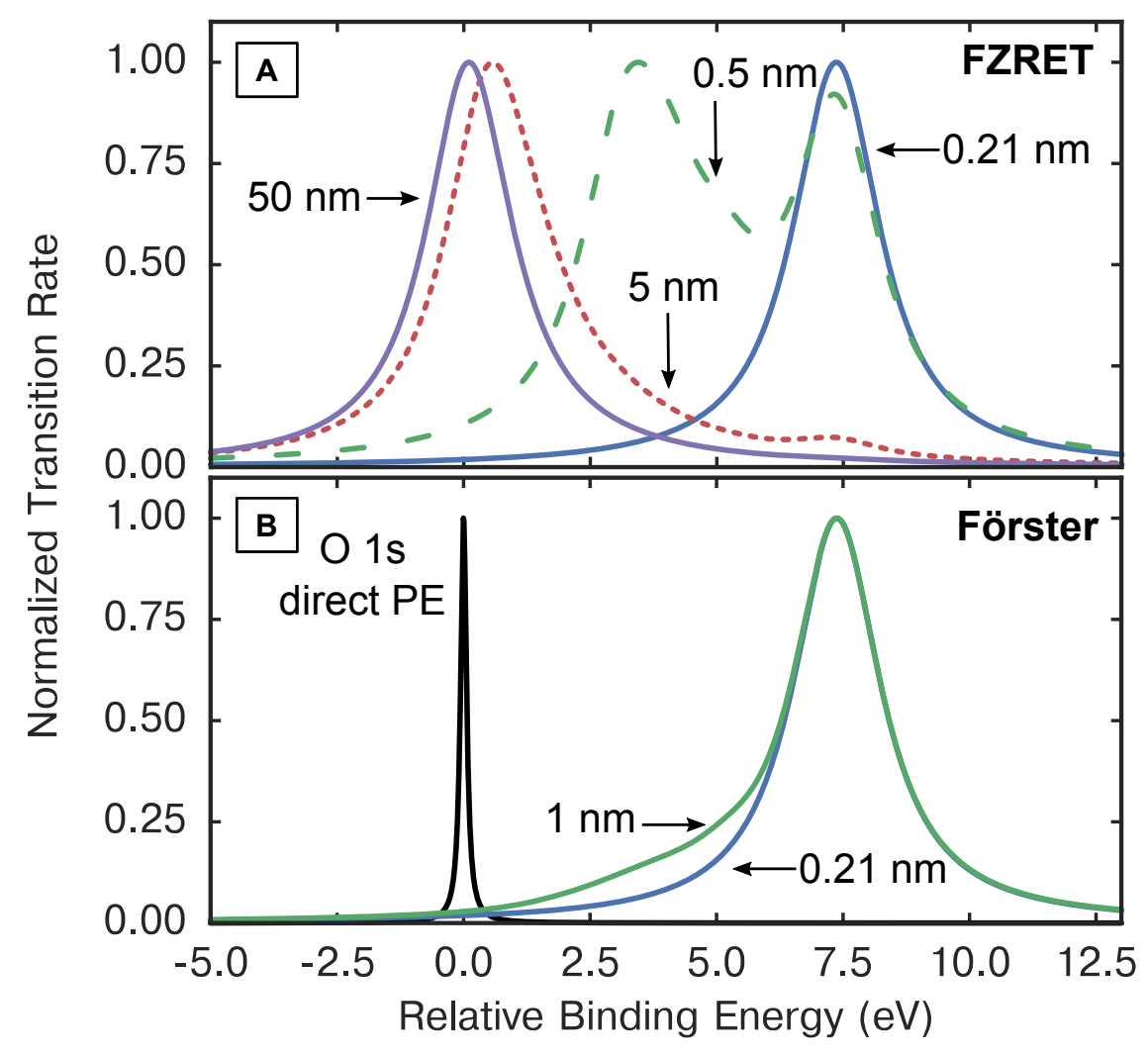

Figure 4: FZRET and Förster RET rate simulations for $\mathrm{CuO}$ crystal. We compute RET profiles for several spherical volumes within the cut-off radius $R_{c}$. (A). FZRET rate (equation 1 with $k=2.15$ a.u.) computed for the cut-off radius $R_{c}$ taken to be $0.21,0.5,5$ and $50 \mathrm{~nm}$. We performed the simulations up to $\mathbf{R}_{c}=100 \mathrm{~nm}$, profile converges at $\mathbf{R}_{c}=50 \mathrm{~nm}$ and shows no shift for $R \geq 50 \mathrm{~nm}$. (B) Förster rate (equation 1 with $k=0$ ) converges at $R_{c}=1 \mathrm{~nm}$ and predicts shifted RET profile. 
considerations (see Supporting Information for details). It is important to note that even though we observe intramolecular RET, it is a far-zone process due to the overwhelmingly large value of the far-zone $(k R)^{4}=13.5$ term in the transfer rate. We also stress that the Förster RET mechanism is completely inadequate in the hard X-ray region.

Comparing the two limiting cases, $\mathrm{CuO}$ polycrystalline powder and $\mathrm{SF}_{6}$ diluted gas, we conclude that the shift observed in a FZRET process can be used for an estimation of the donoracceptor separations in diluted systems up to approximately $5 \mathrm{~nm}$ due to the small size of the shift for large distances. The method is not suitable when there is a high density of acceptors in the far-zone region, which is a typical situation in the condensed phase. The FZRET method can still be applied to condensed phase studies if both donor and acceptor atoms are diluted in a solvent, e.g. to measure separations between different admixtures in doped semiconductor films or distances to ligands in $d$-metal complexes in diluted solution. In this case, the donor-acceptor pairs are identified through their $1 s$ ionization potentials. The upper limit of applicability of this method corresponds to the typical size of globular proteins, and FZRET studies of proteins containing specific donor and acceptor atoms, like iron and sulfur, could provide geometrical estimates. Though the intensity of FZRET X-ray photoemission signals obtained after excitation of the donor by synchrotron radiation is significant, there is still room for improvement of the resolution by using intense and short X-ray free-electron laser pulses. This source also brings an opportunity to map the structural dynamics of the core-ionized systems at the ultrashort timescale, providing a tool similar to time-resolved Förster RET. ${ }^{30}$

In conclusion, we have demonstrated a far-zone resonant energy transfer phenomenon (FZRET) in the X-ray region, and analyzed its characteristics for two different systems - diluted $\mathrm{SF}_{6}$ gas and $\mathrm{CuO}$ polycrystalline powder. Several fundamental differences with the ordinary near-zone resonant energy transfer process in the optical region are unravelled. The use of high energy photons offers distinctive features as compared to optical photons in terms of large penetration depth, chemical selectivity and localization of excitations at the atomic level. The Coulomb shift of the RET resonance of an acceptor induced by core ionization of a donor may in these respects offer an op- 
portunity to explore local structure. In fact, through the application of a simple potential model we argue that the FZRET experiment could be useful as a tool to estimate bond distances and other geometrical information.

\section{Acknowledgement}

Experiments were performed at the GALAXIES beamline at SOLEIL Synchrotron, France (project No. 99130171). The research leading to these results has received funding from the European Union Seventh Framework Programme (FP7/2007-2013) under grant agreement $n^{o} 252781$ and the COST action CM1204 - XUV/x-ray light and fast ions for ultrafast chemistry (XLIC), from Triangle de la Physique under contract 2007-010T, from JSPS and from the Swedish Research Council (VR). We acknowledge financial support from the Knut and Alice Wallenberg Foundation (Grant No. KAW-2013.0020) and Russian Science Foundation (project 16-12-10109).

Supporting Information. Experimental details, analysis of relative contributions from the far-zone RET and computational details are provided in the supporting information.

\section{References}

(1) Scholes, G. D.; Fleming, G. R.; Olaya-Castro, A.; van Grondelle, R. Lessons from nature about solar light harvesting. Nature chemistry 2011, 3, 763-774.

(2) Winkler, J. R. FRETting over the spectroscopic ruler. Science 2013, 339, 1530-1531.

(3) Abramavicius, D.; Mukamel, S. Energy-transfer and charge-separation pathways in the reaction center of photosystem II revealed by coherent two-dimensional optical spectroscopy. The Journal of chemical physics 2010, 133, 184501.

(4) Förster, T. Energiewanderung und fluoreszenz. Naturwissenschaften 1946, 33, 166-175. 
(5) Förster, T. Zwischenmolekulare energiewanderung und fluoreszenz. Annalen der physik 1948, 437, 55-75.

(6) Stryer, L.; Haugland, R. P. Energy transfer: a spectroscopic ruler. Proceedings of the National Academy of Sciences 1967, 58, 719-726.

(7) Avery, J. Resonance energy transfer and spontaneous photon emission. Proceedings of the Physical Society 1966, 88, 1.

(8) Gomberoff, L.; Power, E. A. The resonance transfer of excitation. Proceedings of the Physical Society 1966, 88, 281.

(9) Power, E. A.; Thirunamachandran, T. Quantum electrodynamics with nonrelativistic sources. III. Intermolecular interactions. Physical Review A 1983, 28, 2671-2675.

(10) Andrews, D. L. A unified theory of radiative and radiationless molecular-energy transfer. Chem. Phys. 1989, 135, 195-201.

(11) Andrews, D. L.; Bradshaw, D. S. Virtual photons, dipole fields and energy transfer: a quantum electrodynamical approach. European Journal of Physics 2004, 25, 845-858.

(12) Siegbahn, K.; Nordling, C.; Johansson, G.; Hedman, J.; Hedén, P. F.; Hamrin, K.; Gelius, U.; Bergmark, T.; Werme, L. O.; Manne, R. et al. ESCA applied to free molecules; North-Holland Publishing Company, 1969.

(13) Kay, A.; Arenholz, E.; Mun, S.; de Abajo, F. G.; Fadley, C.; Denecke, R.; Hussain, Z.; Van Hove, M. Multi-atom resonant photoemission: a method for determining near-neighbor atomic identities and bonding. Science 1998, 281, 679-683.

(14) Carravetta, V.; Ågren, H. An ab initio method for computing multi-atom resonant photoemission. Chemical physics letters 2002, 354, 100-108. 
(15) Chantler, C. Theoretical form factor, attenuation, and scattering tabulation for Z=1-92 from $\mathrm{E}=1-10 \mathrm{eV}$ to E=0.4-1.0 MeV. Journal of Physical and Chemical Reference Data 1995, 24, $71-643$.

(16) Chantler, C. Detailed tabulation of atomic form factors, photoelectric absorption and scattering cross section, and mass attenuation coefficients in the vicinity of absorption edges in the soft $X$-ray $(Z=30-36, Z=60-89, E=0.1 \mathrm{keV}-10 \mathrm{keV})$, addressing convergence issues of earlier work. Journal of Physical and Chemical Reference Data 2000, 29, 597-1056.

(17) Gel'mukhanov, F.; Ågren, H. Resonant inelastic x-ray scattering with symmetry-selective excitation. Physical Review A 1994, 49, 4378.

(18) Gel'mukhanov, F.; Ågren, H. Resonant X-ray Raman scattering. Physics Reports 1999, 312, $87-330$.

(19) De Abajo, F. G.; Fadley, C.; Van Hove, M. Multiatom resonant photoemission: theory and systematics. Physical review letters 1999, 82, 4126.

(20) Céolin, D.; Ablett, J.; Prieur, D.; Moreno, T.; Rueff, J.-P.; Marchenko, T.; Journel, L.; Guillemin, R.; Pilette, B.; Marin, T. et al. Hard X-ray photoelectron spectroscopy on the GALAXIES beamline at the SOLEIL synchrotron. Journal of Electron Spectroscopy and Related Phenomena 2013, 190, 188-192.

(21) Rueff, J.-P.; Ablett, J.; Céolin, D.; Prieur, D.; Moreno, T.; Balédent, V.; Lassalle-Kaiser, B.; Rault, J.; Simon, M.; Shukla, A. The GALAXIES beamline at the SOLEIL synchrotron: inelastic X-ray scattering and photoelectron spectroscopy in the hard X-ray range. Journal of synchrotron radiation 2015, 22, 175-179.

(22) Kuchiev, M.; Shel̆nerman, S. Post-collision interaction in atomic processes. Physics-Uspekhi 1989, 32, 569-587. 
(23) Kraft, S.; Stümpel, J.; Becker, P.; Kuetgens, U. High resolution x-ray absorption spectroscopy with absolute energy calibration for the determination of absorption edge energies. Review of scientific instruments 1996, 67, 681-687.

(24) Hölzer, G.; Fritsch, M.; Deutsch, M.; Härtwig, J.; Förster, E. K $\alpha 1,2$ and K $\beta 1,3$ X-ray emission lines of the 3d transition metals. Physical Review A 1997, 56, 4554.

(25) Reynaud, C.; Bodeur, S.; Maréchal, J.; Bazin, D.; Millié, P.; Nenner, I.; Rockland, U.; Baumgärtel, $\mathrm{H}$. Electronic properties of the $\mathrm{SF}_{5} \mathrm{Cl}$ molecule: a comparison with $\mathrm{SF}_{6}$. I. Photoabsorption spectra near the sulphur K and chlorine K edges. Chemical physics 1992, 166, $411-424$.

(26) Piancastelli, M. N.; Stolte, W. C.; Guillemin, R.; Wolska, A.; Yu, S.-W.; Santanna, M.; Lindle, D. Anion and cation-yield spectroscopy of core-excited $\mathrm{SF}_{6}$. The Journal of chemical physics 2005, 122, 094312.

(27) LaVilla, R. E. The Sulfur K and L and Fluorine K X-Ray Emission and Absorption Spectra of Gaseous $\mathrm{SF}_{6}$. The Journal of Chemical Physics 1972, 57, 899-909.

(28) Hayashi, H.; Udagawa, Y.; Caliebe, W.; Kao, C.-C. Hidden electronic state of CuO revealed by resonant inelastic x-ray scattering. Physical Review B 2002, 66, 033105.

(29) Bartell, L.; Doun, S. Structures of hexacoordinate compounds of main-group elements: Part III. An electron diffraction study of $\mathrm{SF}_{6}$. Journal of Molecular Structure 1978, 43, 245-249.

(30) Geißler, D.; Stufler, S.; Löhmannsröben, H.-G.; Hildebrandt, N. Six-Color Time-Resolved Förster Resonance Energy Transfer For Ultrasensitive Multiplexed Biosensing. Journal of the American Chemical Society 2012, 135, 1102-1109. 